Research Article

\title{
The Averaging Principle for Stochastic Pantograph Equations with Non-Lipschitz Conditions
}

\author{
Dongdong Gao $\mathbb{D},{ }^{1}$ Jianli Li $\mathbb{D},{ }^{2}$ Zhiguo Luo $\mathbb{D}^{2},{ }^{2}$ and Danfeng Luo $\mathbb{D}^{3}$ \\ ${ }^{1}$ Department of Mathematics and Computer Science, Tongling University, Tongling, Anhui 244000, China \\ ${ }^{2}$ College of Mathematics and Statistics, Hunan Normal University, Changsha, Hunan 410081, China \\ ${ }^{3}$ Department of Mathematics, Guizhou University, Guiyang, Guizhou 550025, China
}

Correspondence should be addressed to Danfeng Luo; luodf0916@sohu.com

Received 25 January 2021; Revised 22 March 2021; Accepted 8 April 2021; Published 21 April 2021

Academic Editor: Francesco Riganti-Fulginei

Copyright (c) 2021 Dongdong Gao et al. This is an open access article distributed under the Creative Commons Attribution License, which permits unrestricted use, distribution, and reproduction in any medium, provided the original work is properly cited.

This paper is devoted to presenting an averaging principle for stochastic pantograph equations. Under suitable non-Lipschitz conditions, the solutions to stochastic pantograph equations can be approximated by solutions to averaged stochastic systems in the mean-square sense and probability. At last, an example is given to demonstrate the feasibility of obtained results. Moreover, our results have generalized significantly some previous ones.

\section{Introduction}

Pantograph equations [1] are a kind of equations with unbounded delay, and they were used in describing the various phenomena like biology, electrodynamics, economy, and some other nonlinear dynamical systems [2-4]. Based on these irreplaceable roles, the existence, uniqueness, and stability for different kinds of pantograph equations were tremendously investigated by many scholars. Of course, some excellent and important articles have also emerged in our vision (see [5-8] and references therein).

On the contrary, the averaging principle as a powerful method has been largely applied in stochastic differential systems, and its main role is to strike a balance between complex models that are more realistic and simpler models that are more amenable to analysis and simulation. Referring to the averaging principle, we are indispensable to recall some excellent articles [9-13], which have discussed the corresponding solutions to stochastic differential equations by the averaging principle.

To the best of authors' knowledge, there is no paper which has considered the approximation theorem as an averaging principle for stochastic pantograph equations. To fill this gap, in this paper, we are intended to study the stochastic pantograph equations as follows:

$$
\left\{\begin{array}{l}
d X(t)=f(t, X(t), X(\theta t)) \mathrm{d} t+g(t, X(t), X(\theta t)) \mathrm{d} B(t) \\
X(0)=X_{0}
\end{array}\right.
$$

where $t \in[0, T], X_{0} \in \mathbb{R}^{n}$ is the initial value, which is $\mathscr{F}_{0}$-measurable on $\mathbb{R}^{n}$ and satisfying $\mathbb{E}\left|X_{0}\right|^{2}<\infty, 0<\theta<1$, $f:[0, T] \times \mathbb{R}^{n} \times \mathbb{R}^{n} \longrightarrow \mathbb{R}^{n}, g:[0, T] \times \mathbb{R}^{n} \times \mathbb{R}^{n} \longrightarrow \mathbb{R}^{n \times m}$, and $B(t)$ is a $m$-dimensional Brownian motion on the complete probability space $(\Omega, \mathscr{F}, \mathbb{P})$.

The highlights and major contributions of this paper are reflected in the subsequent key aspects:

(i) We first attempt to investigate the property of solutions for a class of stochastic pantograph equations by the averaging principle under the non-Lipschitz conditions. Comparing with some previous literatures [5-8], the corresponding conditions are required to satisfy the Lipschitz condition or the local Lipschitz condition. However, in some practical cases, the Lipschitz condition is usually violated. Therefore, the Lipschitz condition will be replaced by the non-Lipschitz conditions which are much weaker than the Lipschitz condition in our paper.

(ii) The previous literatures [9-11] have not considered the effect of delay terms on the averaging principle 
for corresponding stochastic system. However, the delay effects do exist in these stochastic differential systems. Therefore, in this paper, we consider a kind of delay stochastic differential equations with a linear delay $\tau(t)=\theta t$ with $0<\theta<1$.

The arrangement of the rest paper is as follows. In Section 2, necessary definition and hypothesis conditions will be presented. Section 3 is devoted to present the averaging principle for stochastic pantograph equation (1). In Section 4, we will give an example to demonstrate the feasibility of theoretical results obtained in Section 3.

\section{Preliminaries}

In this section, we will give some necessary definition and hypothesis conditions, which will be used in later sections.

Definition 1. An $\mathbb{R}^{n}$-value stochastic process $\{X(t)\}_{0 \leq t \leq T}$ is called a unique solution of (1) if the following conditions hold:

(i) $\{X(t)\}$ is continuous with respect to $t$ and $\mathscr{F}_{t}$ adapted.

(ii) $f(t, X(t), X(\theta t)) \in \mathscr{L}^{1}\left([0, T] ; \mathbb{R}^{n}\right)$ and $g(t, X(t)$, $X(\theta t)) \in \mathscr{L}^{2}\left([0, T] ; \mathbb{R}^{n \times m}\right)$.

(iii) For all $t \in[0, T]$, we have

$$
X(t)=X_{0}+\int_{0}^{t} f(s, X(s), X(\theta s)) \mathrm{d} s+\int_{0}^{t} g(s, X(s), X(\theta s)) \mathrm{d} B(s), \text { a.s. }
$$

(iv) For any other solution $X^{*}(t)$, we have $P\left\{X(t)=X^{*}(t), \forall t \in[0, T]\right\}=1$.

Next, we list the necessary hypothesis conditions, which will be used to prove the main results in next section.
(H1) (non-Lipschitz condition): there exists a function $\Phi(\cdot)$ such that, for any fixed $t \geq 0$ and $X_{i}, Y_{i} \in R^{n}, i=1,2$, we have

$$
\left|f\left(t, X_{1}, Y_{1}\right)-f\left(t, X_{2}, Y_{2}\right)\right|^{2} \vee\left|g\left(t, X_{1}, Y_{1}\right)-g\left(t, X_{2}, Y_{2}\right)\right|^{2} \leq \Phi\left(t,\left|X_{1}-X_{2}\right|^{2},\left|Y_{1}-Y_{2}\right|^{2}\right),
$$

where $\Phi(\cdot)$ satisfies $\Phi(t, 0,0)=0$ and define $\Omega=[0,+\infty) \times$ $[0,+\infty)$ and $\int_{\Omega}(1 / \Phi(t, u, v)) \mathrm{d} u \mathrm{~d} v=\infty$ and there exist nonnegative functions $\lambda_{i}(t), i=1,2,3$ such that, for $u, v \geq 0$, $\Phi(t, u, v) \leq \lambda_{1}(t)+\lambda_{2}(t) u+\lambda_{3}(t) v$ $\int_{0}^{T} \lambda_{i}(t) \mathrm{d} t<\infty, i=1,2,3$.

\section{Main Results}

This section is devoted to present an averaging principle for stochastic pantograph equations. First, we consider the standard form of the system (1):

$$
X_{\varepsilon}(t)=X_{0}+\varepsilon \int_{0}^{t} f\left(s, X_{\varepsilon}(s), X_{\varepsilon}(\theta s)\right) \mathrm{d} s+\sqrt{\varepsilon} \int_{0}^{t} g\left(s, X_{\varepsilon}(s), X_{\varepsilon}(\theta s)\right) \mathrm{d} B(s),
$$

where $\varepsilon \in\left(0, \varepsilon_{0}\right]$ is a positive small parameter and $\varepsilon_{0}$ is a given fixed number.

To obtain the averaging principle for stochastic pantograph equations, we also need following condition: there exist measurable functions $f^{*}: \mathbb{R}^{n} \times \mathbb{R}^{n} \longrightarrow \mathbb{R}^{n}$ and $g^{*}: \mathbb{R}^{n} \times \mathbb{R}^{n} \longrightarrow \mathbb{R}^{n \times m}$ such that the following holds.

(H2): for any $T_{1} \in[0, T], X, Y \in \mathbb{R}^{n}$, there exist two positive bounded functions $\Psi_{i}\left(T_{1}\right), i=1,2$, such that

$$
\begin{gathered}
\frac{1}{T_{1}} \int_{0}^{T_{1}}\left|f(s, X, Y)-f^{*}(X, Y)\right|^{2} \mathrm{~d} s \leq \Psi_{1}\left(T_{1}\right)\left(|X|^{2}+|Y|^{2}\right), \\
\frac{1}{T_{1}} \int_{0}^{T_{1}}\left|g(s, X, Y)-g^{*}(X, Y)\right|^{2} \mathrm{~d} s \leq \Psi_{2}\left(T_{1}\right)\left(|X|^{2}+|Y|^{2}\right),
\end{gathered}
$$

where $\lim _{T_{1} \rightarrow \infty} \Psi_{i}\left(T_{1}\right)=0$.

By the above preparation, we now consider the original solution $X_{\varepsilon}(t)$ converges to the solution $X_{\varepsilon}^{*}(t)$ of the averaged system:

$$
\begin{aligned}
X_{\varepsilon}^{*}(t)= & X_{0}+\varepsilon \int_{0}^{t} f^{*}\left(X_{\varepsilon}^{*}(s), X_{\varepsilon}^{*}(\theta s)\right) \mathrm{d} s \\
& +\sqrt{\varepsilon} \int_{0}^{t} g^{*}\left(X_{\varepsilon}^{*}(s), X_{\varepsilon}^{*}(\theta s)\right) \mathrm{d} B(s),
\end{aligned}
$$

as $\varepsilon$ goes to zero and $t \in[0, T]$.

Theorem 1. Assume that conditions (H1) and (H2) hold. Then, for a given arbitrarily small number $\delta_{1}>0$, there exist $M>0, \varepsilon_{1} \in\left(0, \varepsilon_{0}\right]$ and $\beta \in(0,1)$ such that, for all $\varepsilon \in\left(0, \varepsilon_{1}\right]$, we have

$$
\mathbb{E}\left(\sup _{t \in\left[0, M \varepsilon^{-\beta}\right]}\left|X_{\varepsilon}(t)-X_{\varepsilon}^{*}(t)\right|^{2}\right) \leq \delta_{1} .
$$

Proof. For any $t \in[0, u] \subset[0, T]$, by (4) and (6), we have 


$$
\begin{aligned}
X_{\varepsilon}(t) & -X_{\varepsilon}^{*}(t)=\varepsilon \int_{0}^{t}\left(f\left(s, X_{\varepsilon}(s), X_{\varepsilon}(\theta s)\right)-f^{*}\left(X_{\varepsilon}^{*}(s), X_{\varepsilon}^{*}(\theta s)\right)\right) \mathrm{d} s \\
& +\sqrt{\varepsilon} \int_{0}^{t}\left(g\left(s, X_{\varepsilon}(s), X_{\varepsilon}(\theta s)\right)-g^{*}\left(X_{\varepsilon}^{*}(s), X_{\varepsilon}^{*}(\theta s)\right)\right) \mathrm{d} B(s) .
\end{aligned}
$$

Taking mathematical expectation on above equation and applying the elementary inequality, we have

$$
\begin{aligned}
\mathbb{E}\left(\sup _{0 \leq t \leq \mathcal{u}}\left|X_{\varepsilon}(t)-X_{\varepsilon}^{*}(t)\right|^{2}\right) \leq & 2 \varepsilon^{2} \mathbb{E} \sup _{0 \leq t \leq u}\left|\int_{0}^{t} f\left(s, X_{\varepsilon}(s), X_{\varepsilon}(\theta s)-f^{*} X_{\varepsilon}^{*}(s), X_{\varepsilon}^{*}(\theta s)\right) \mathrm{d} s\right|^{2} \\
& +2 \varepsilon \mathbb{E} \sup _{0 \leq t \leq u}\left|\int_{0}^{t} g\left(s, X_{\varepsilon}(s), X_{\varepsilon}(\theta s)-g^{*} X_{\varepsilon}^{*}(s), X_{\varepsilon}^{*}(\theta s)\right) \mathrm{d} B(s)\right|^{2} \\
= & I_{1}+I_{2} .
\end{aligned}
$$

Using the elementary inequality again, we have

$$
\begin{aligned}
I_{1} \leq & 4 \varepsilon^{2} \mathbb{E} \sup _{0 \leq t \leq u}\left|\int_{0}^{t}\left(f\left(s, X_{\varepsilon}(s), X_{\varepsilon}(\theta s)\right)-f\left(s, X_{\varepsilon}^{*}(s), X_{\varepsilon}^{*}(\theta s)\right)\right) \mathrm{d} s\right|^{2} \\
& +4 \varepsilon^{2} \mathbb{E} \sup _{0 \leq t \leq u}\left|\int_{0}^{t}\left(f\left(s, X_{\varepsilon}^{*}(s), X_{\varepsilon}^{*}(\theta s)\right)-f^{*}\left(X_{\varepsilon}^{*}(s), X_{\varepsilon}^{*}(\theta s)\right)\right) \mathrm{d} s\right|^{2} \\
= & I_{11}+I_{12} .
\end{aligned}
$$

From (H1) and Cauchy-Schwarz inequality, we have

$$
\begin{aligned}
I_{11} \leq & 4 \varepsilon^{2} u \mathbb{E} \sup _{0 \leq t \leq u} \int_{0}^{t}\left|f\left(s, X_{\varepsilon}(s), X_{\varepsilon}(\theta s)\right)-f\left(s, X_{\varepsilon}^{*}(s), X_{\varepsilon}^{*}(\theta s)\right)\right|^{2} \mathrm{~d} s \\
\leq & 4 \varepsilon^{2} u \mathbb{E} \sup _{0 \leq t \leq u} \int_{0}^{t} \Phi\left(s,\left|X_{\varepsilon}(s)-X_{\varepsilon}^{*}(s)\right|^{2},\left|X_{\varepsilon}(\theta s)-X_{\varepsilon}^{*}(\theta s)\right|^{2}\right) \mathrm{d} s \\
\leq & 4 \varepsilon^{2} u \mathbb{E} \sup _{0 \leq t \leq u} \int_{0}^{t}\left(\lambda_{1}(s)+\lambda_{2}(s)\left|X_{\varepsilon}(s)-X_{\varepsilon}^{*}(s)\right|^{2}+\lambda_{3}(s)\left|X_{\varepsilon}(\theta s)-X_{\varepsilon}^{*}(\theta s)\right|^{2}\right) \mathrm{d} s \\
\leq & 4 \varepsilon^{2} u\left[u \sup _{0 \leq t \leq u} \lambda_{1}(t)+\mathbb{E} \sup _{0 \leq t \leq u} \int_{0}^{t}\left(\lambda_{2}(s)\left|X_{\varepsilon}(s)-X_{\varepsilon}^{*}(s)\right|^{2}\right.\right. \\
& \left.\left.+\lambda_{3}(s)\left|X_{\varepsilon}(\theta s)-X_{\varepsilon}^{*}(\theta s)\right|^{2}\right) \mathrm{~d} s\right] \\
\leq & 4 \varepsilon^{2} u^{2} \sup _{0 \leq t \leq u} \lambda_{1}(t)+4 \varepsilon^{2} u \sum_{i=2}^{3} \sup _{0 \leq t \leq u} \lambda_{i}(t) \int_{0}^{u} \mathbb{E} \sup _{0 \leq s_{1} \leq s}\left|X_{\varepsilon}\left(s_{1}\right)-X_{\varepsilon}^{*}\left(s_{1}\right)\right|^{2} \mathrm{~d} s .
\end{aligned}
$$


Also, from (H2) and Cauchy-Schwarz inequality, we have

$$
\begin{aligned}
I_{12} & \leq 4 \varepsilon^{2} u \mathbb{E} \sup _{0 \leq t \leq u} \int_{0}^{t}\left|\left(f\left(s, X_{\varepsilon}^{*}(s), X_{\varepsilon}^{*}(\theta s)\right)-f^{*}\left(X_{\varepsilon}^{*}(s), X_{\varepsilon}^{*}(\theta s)\right)\right)\right|^{2} \mathrm{~d} s \\
& \leq 4 \varepsilon^{2} u^{2} \mathbb{E} \sup _{0 \leq t \leq u} \frac{1}{t} \int_{0}^{t}\left|\left(f\left(s, X_{\varepsilon}^{*}(s), X_{\varepsilon}^{*}(\theta s)\right)-f^{*}\left(X_{\varepsilon}^{*}(s), X_{\varepsilon}^{*}(\theta s)\right)\right)\right|^{2} \mathrm{~d} s \\
& \leq 4 \varepsilon^{2} u^{2}\left(\sup _{0 \leq t \leq u} \Psi_{1}(t)\right) \mathbb{E}\left[\left(\sup _{0 \leq t \leq u}\left|X_{\varepsilon}^{*}(t)\right|^{2}\right)+\left(\sup _{0 \leq t \leq u}\left|X_{\varepsilon}^{*}(\theta t)\right|^{2}\right)\right] \\
& \leq 8 \varepsilon^{2} u^{2}\left(\sup _{0 \leq t \leq u} \Psi_{1}(t)\right) \mathbb{E}\left[\left(\sup _{0 \leq t \leq u}\left|X_{\varepsilon}^{*}(t)\right|^{2}\right)\right] .
\end{aligned}
$$
have

Analogously, by using the elementary inequality, we also

$$
\begin{aligned}
I_{2} \leq & 4 \varepsilon \mathbb{E} \sup _{0 \leq t \leq u}\left|\int_{0}^{t}\left(g\left(s, X_{\varepsilon}(s), X_{\varepsilon}(\theta s)\right)-\operatorname{tgn}\left(s, X_{\varepsilon}^{*}(s), X_{\varepsilon}^{*}(\theta s)\right)\right) \mathrm{d} B(s)\right|^{2} \\
& +4 \varepsilon \mathbb{E} \sup _{0 \leq t \leq u}\left|\int_{0}^{t}\left(g\left(s, X_{\varepsilon}^{*}(s), X_{\varepsilon}^{*}(\theta s)\right)-\operatorname{tg}^{*} n\left(X_{\varepsilon}^{*}(s), X_{\varepsilon}^{*}(\theta s)\right)\right) \mathrm{d} B(s)\right|^{2} \\
= & I_{21}+I_{22} .
\end{aligned}
$$

From (H1) and Burkholder-Davis-Gundy inequality, we have

$$
\begin{aligned}
I_{21} & \leq 16 \varepsilon \mathbb{E} \sup _{0 \leq t \leq u}\left|\int_{0}^{t}\left(g\left(s, X_{\varepsilon}(s), X_{\varepsilon}(\theta s)\right)-g\left(s, X_{\varepsilon}^{*}(s), X_{\varepsilon}^{*}(\theta s)\right)\right) \mathrm{d} s\right|^{2} \\
& \leq 16 \varepsilon u \sup _{0 \leq t \leq u} \int_{0}^{t}\left|g\left(s, X_{\varepsilon}(s), X_{\varepsilon}(\theta s)\right)-g\left(s, X_{\varepsilon}^{*}(s), X_{\varepsilon}^{*}(\theta s)\right)\right|^{2} \mathrm{~d} s \\
& \leq 16 \varepsilon u \mathbb{E} \sup _{0 \leq t \leq u} \int_{0}^{t} \Phi\left(s,\left|X_{\varepsilon}(s)-X_{\varepsilon}^{*}(s)\right|^{2},\left|X_{\varepsilon}(\theta s)-X_{\varepsilon}^{*}(\theta s)\right|^{2}\right) \mathrm{d} s \\
& \leq 16 \varepsilon u \mathbb{E} \sup _{0 \leq t \leq u} \int_{0}^{t}\left(\lambda_{1}(s)+\lambda_{2}(s)\left|X_{\varepsilon}(s)-X_{\varepsilon}^{*}(s)\right|^{2}+\lambda_{3}(s)\left|X_{\varepsilon}(\theta s)-X_{\varepsilon}^{*}(\theta s)\right|^{2}\right) \mathrm{d} s \\
& \leq 16 \varepsilon u\left[u \sup _{0 \leq t \leq u} \lambda_{1}(t)+\mathbb{E} \sup _{0 \leq t \leq u}^{t} \int_{0}^{t}\left(\lambda_{2}(s)\left|X_{\varepsilon}(s)-X_{\varepsilon}^{*}(s)\right|^{2}\right.\right. \\
& \left.\left.+\lambda_{3}(s)\left|X_{\varepsilon}(\theta s)-X_{\varepsilon}^{*}(\theta s)\right|^{2}\right) \mathrm{~d} s\right] \\
& \leq 16 \varepsilon u^{2} \sup _{0 \leq t \leq u} \lambda_{1}(t)+16 \varepsilon u \sum_{i=2}^{3} \sup _{0 \leq t \leq u} \lambda_{i}(t) \int_{0}^{u} \mathbb{E} \sup _{0 \leq s_{1} \leq s}\left|X_{\varepsilon}\left(s_{1}\right)-X_{\varepsilon}^{*}\left(s_{1}\right)\right|^{2} \mathrm{~d} s .
\end{aligned}
$$


Also, from (H2) and Burkholder-Davis-Gundy inequality, we have

$$
\begin{aligned}
I_{22} & \leq 16 \varepsilon \mathbb{E} \sup _{0 \leq t \leq u}\left|\int_{0}^{t}\left(g\left(s, X_{\varepsilon}^{*}(s), X_{\varepsilon}^{*}(\theta s)\right)-g^{*}\left(X_{\varepsilon}^{*}(s), X_{\varepsilon}^{*}(\theta s)\right)\right) \mathrm{d} s\right|^{2} \\
& \leq 16 \varepsilon u^{2} \mathbb{E} \sup _{0 \leq t \leq u} \frac{1}{t} \int_{0}^{t}\left|\left(g\left(s, X_{\varepsilon}^{*}(s), X_{\varepsilon}^{*}(\theta s)\right)-g^{*}\left(X_{\varepsilon}^{*}(s), X_{\varepsilon}^{*}(\theta s)\right)\right)\right|^{2} \mathrm{~d} s \\
& \leq 16 \varepsilon u^{2}\left(\sup _{0 \leq t \leq u} \Psi_{2}(t)\right) \mathbb{E}\left[\left(\sup _{0 \leq t \leq u}\left|X_{\varepsilon}^{*}(t)\right|^{2}\right)+\left(\sup _{0 \leq t \leq u}\left|X_{\varepsilon}^{*}(\theta t)\right|^{2}\right)\right] \\
& \leq 32 \varepsilon u^{2}\left(\sup _{0 \leq t \leq u} \Psi_{2}(t)\right) \mathbb{E}\left[\left(\sup _{0 \leq t \leq u}\left|X_{\varepsilon}^{*}(t)\right|^{2}\right)\right] .
\end{aligned}
$$

According to the estimation of $I_{11}, I_{12}, I_{21}$, and $I_{22}$, we have

$$
\begin{aligned}
\mathbb{E}\left(\sup _{0 \leq t \leq u}\left|X_{\varepsilon}(t)-X_{\varepsilon}^{*}(t)\right|^{2}\right) \\
\leq 4 \varepsilon^{2} u^{2} \sup _{0 \leq t \leq u} \lambda_{1}(t)+16 \varepsilon u^{2} \sup _{0 \leq t \leq u} \lambda_{1}(t) \\
+8 \varepsilon^{2} u^{2}\left(\sup _{0 \leq t \leq u} \Psi_{1}(t)\right) \mathbb{E}\left[\left(\sup _{0 \leq t \leq u}\left|X_{\varepsilon}^{*}(t)\right|^{2}\right)\right] \\
+32 \varepsilon u^{2}\left(\sup _{0 \leq t \leq u} \Psi_{2}(t)\right) \mathbb{E}\left[\left(\sup _{0 \leq t \leq u}\left|X_{\varepsilon}^{*}(t)\right|^{2}\right)\right] \\
\quad+4 \varepsilon^{2} u \sum_{i=2}^{3} \sup _{0 \leq t \leq u} \lambda_{i}(t) \int_{0}^{u} \mathbb{E} \sup _{0 \leq s_{1} \leq s}\left|X_{\varepsilon}\left(s_{1}\right)-X_{\varepsilon}^{*}\left(s_{1}\right)\right|^{2} \mathrm{~d} s \\
\quad+16 \varepsilon u \sum_{i=2}^{3} \sup _{0 \leq t \leq u} \lambda_{i}(t) \int_{0}^{u} \mathbb{E} \sup _{0 \leq s_{1} \leq s}\left|X_{\varepsilon}\left(s_{1}\right)-X_{\varepsilon}^{*}\left(s_{1}\right)\right|^{2} \mathrm{~d} s \\
\leq 4 M_{\lambda} \varepsilon^{2} u^{2}+16 M_{\lambda} \varepsilon u^{2}+8 M_{\Psi} \varepsilon^{2} u^{2}+32 M_{\Psi} \varepsilon u^{2} \\
+\left(8 \varepsilon^{2} u+32 \varepsilon u\right) \int_{0}^{u} \mathbb{E} \sup _{0 \leq s_{1} \leq s}\left|X_{\varepsilon}\left(s_{1}\right)-X_{\varepsilon}^{*}\left(s_{1}\right)\right|^{2} \mathrm{~d} s
\end{aligned}
$$

where $\left\{\sup _{0 \leq t \leq u} \Psi_{1}(t) \mathbb{E}\left[\left(\sup _{0 \leq t \leq u}\left|X_{\varepsilon}^{*}(t)\right|^{2}\right)\right], \sup _{0 \leq t \leq u} \Psi_{2}\right.$ $\left.(t) \mathbb{E}\left[\left(\sup _{0 \leq t \leq u}\left|X_{\varepsilon}^{*}(t)\right|^{2}\right)\right]\right\}=M_{\Psi}$, and $\left\{\sup _{0 \leq t \leq u} \lambda_{i}(t)\right.$, $i=1,2,3\}=M_{\lambda}$. With the help of the Gronwall-Bellman inequality, we get

$$
\mathbb{E}\left(\sup _{0 \leq t \leq u}\left|X_{\varepsilon}(t)-X_{\varepsilon}^{*}(t)\right|^{2}\right) \leq\left(4 M_{\lambda} \varepsilon^{2} u^{2}+16 M_{\lambda} \varepsilon u^{2}+8 M_{\Psi} \varepsilon^{2} u^{2}+32 M_{\Psi} \varepsilon u^{2}\right) e^{\left(8 \varepsilon^{2} u^{2}+32 \varepsilon u^{2}\right)}
$$

which implies that there exists a $M>0$ and $\beta \in(0,1)$ such where that for any $t \in\left[0, M \varepsilon^{-\beta}\right] \subset[0, T]$ having

$$
\mathbb{E}\left(\sup _{t \in\left[0, M \varepsilon^{-\beta}\right]}\left|X_{\varepsilon}(t)-X_{\varepsilon}^{*}(t)\right|^{2}\right) \leq K \varepsilon^{1-\beta}
$$

$$
\left.K=\left(4 M_{\lambda} M^{2} \varepsilon^{1-\beta}+16 M_{\lambda} M^{2} \varepsilon^{-\beta}+8 M_{\Psi} M^{2} \varepsilon^{1-\beta}+32 M_{\Psi} M^{2} \varepsilon^{-\beta}\right) e^{\left(8 M^{2} \varepsilon^{2-2 \beta}+32 M^{2} \varepsilon^{1-2 \beta}\right.}\right)
$$


is a constant. As a result, for arbitrary given number $\delta_{1}$, there exists $\varepsilon_{1} \in\left(0, \varepsilon_{0}\right]$ such that, for each $\varepsilon \in\left(0, \varepsilon_{1}\right]$ and $t \in\left[0, M \varepsilon^{-\beta}\right]$, one has

$$
\mathbb{E}\left(\sup _{t \in\left[0, M \varepsilon^{-\beta}\right]}\left|X_{\varepsilon}(t)-X_{\varepsilon}^{*}(t)\right|^{2}\right) \leq \delta_{1} .
$$

The proof is completed.

Next, we will present the properties of the uniform convergence in probability between (4) and (6).

Theorem 2. Assume that conditions (H1) and (H2) hold. Then, for a given arbitrarily small number $\delta_{2}>0$, there exist $M>0, \varepsilon_{1} \in\left(0, \varepsilon_{0}\right]$ and $\beta \in(0,1)$, such that, for all $\varepsilon \in\left(0, \varepsilon_{1}\right]$, we have

$$
\lim _{\varepsilon \longrightarrow 0} \mathbb{P}\left(\sup _{t \in\left[0, M \varepsilon^{-\beta}\right]}\left|X_{\varepsilon}(t)-X_{\varepsilon}^{*}(t)\right|>\delta_{2}\right)=0 .
$$

Proof. Given $\delta_{2}>0$, with the help of Chebyshev's inequality and Theorem 1 , we obtain

$$
\begin{aligned}
& \mathbb{P}\left(\sup _{t \in\left[0, M \varepsilon^{-\beta}\right]}\left|X_{\varepsilon}(t)-X_{\varepsilon}^{*}(t)\right|>\delta_{2}\right) \\
& \leq \frac{1}{\delta_{2}^{2}} \mathbb{E}\left(\sup _{t \in\left[0, M \varepsilon^{-\beta}\right]}\left|X_{\varepsilon}(t)-X_{\varepsilon}^{*}(t)\right|^{2}\right) \\
& \leq \frac{1}{\delta_{2}^{2}} K \varepsilon^{1-\beta} \longrightarrow 0, \quad \text { as } \varepsilon \longrightarrow 0 .
\end{aligned}
$$

The proof is completed.

Remark 1. If $\Phi(t, u, v)=K(u+v)$, the non-Lipschitz conditions reduce to a global Lipschitz conditions which have been investigated in previous literatures [5-8]. So, some previous results [5-8] will be significantly improved in our paper. Also, the averaging principle is applied to study stochastic pantograph equations for the first time. In view of the previous literatures $[14,15]$, the authors have studied the stochastic pantograph equations driven by Lévy noise and G-Brownian motion. To the best of our knowledge, the averaging principle can also use to study stochastic pantograph equations driven by Lévy noise and G-Brownian motion with the non-Lipschitz conditions.

\section{Example}

Consider the following stochastic pantograph equation:

$$
\mathrm{d} X_{\varepsilon}(t)=\varepsilon\left(2 X_{\varepsilon} \cos ^{2}(t)-X_{\varepsilon} \sin ^{2}\left(\frac{1}{2} t\right)\right) \mathrm{d} t+\sqrt{\varepsilon} \mathrm{d} B(t)
$$

where

$$
\begin{gathered}
X_{\varepsilon}=X_{0}, \\
t \in[0, \pi], \\
f\left(t, X_{\varepsilon}(t), X_{\varepsilon}(\theta t)\right)=2 X_{\varepsilon} \cos ^{2}(t)-X_{\varepsilon} \sin ^{2}\left(\frac{1}{2} t\right), \\
g\left(t, X_{\varepsilon}(t), X_{\varepsilon}(\theta t)\right)=1 . \\
\text { Let } g^{*}\left(t, X_{\varepsilon}(t), X_{\varepsilon}(\theta t)\right)=1, \text { and } \\
f^{*}\left(t, X_{\varepsilon}(t), X_{\varepsilon}(\theta t)\right)=\frac{1}{\pi} \int_{0}^{\pi} f\left(t, X_{\varepsilon}(t), X_{\varepsilon}(\theta t)\right)=\frac{X_{\varepsilon}}{2} .
\end{gathered}
$$

Therefore, the simplified stochastic pantograph equation (23) can be defined as

$$
\mathrm{d} X_{\varepsilon}^{*}(t)=\varepsilon \frac{X_{\varepsilon}^{*}}{2} \mathrm{~d} t+\sqrt{\varepsilon} \mathrm{d} B(t) .
$$

It is clear to see all conditions (H1) and (H2) hold for the functions defined in systems (23) and (26). By the Theorems 1 and 2 , we obtain

$$
\begin{array}{r}
\mathbb{E}\left(\sup _{t \in\left[0, M \varepsilon^{-\beta}\right]}\left|X_{\varepsilon}(t)-X_{\varepsilon}^{*}(t)\right|^{2}\right) \leq \delta_{1}, \\
\lim _{\varepsilon \longrightarrow 0} \mathbb{P}\left(\sup _{t \in\left[0, M \varepsilon^{-\beta}\right]}\left|X_{\varepsilon}(t)-X_{\varepsilon}^{*}(t)\right|>\delta_{2}\right)
\end{array}
$$

\section{Conclusion}

In this paper, we mainly discuss an averaging principle for stochastic pantograph equations at the first time. Under suitable non-Lipschitz conditions, we obtain that the solutions to stochastic pantograph equations can be approximated by solutions to averaged stochastic systems in the mean-square sense and probability. Then, an example is given to demonstrate the feasibility of obtained results.

In future work, the interesting extension of our study would be to discuss the averaging principle for the impulsive stochastic pantograph equations with time delays and the fractional stochastic pantograph equations.

\section{Data Availability}

The data in this study were mainly collected via discussion during our class. Readers wishing to access these data can do so by contacting the corresponding author.

\section{Conflicts of Interest}

The authors declare that they have no conflicts of interest.

\section{Acknowledgments}

This work was supported by the NSFC (Grant nos. 12071105 and 11571088) and the Natural Science Special Research Fund Project of Guizhou University (Grant no. 202002). 


\section{References}

[1] J. Ockendon and A. Taylor, "The dynamics of a current collection system for an electric locomotive," Proceedings of the Royal Society London, vol. 322, pp. 447-468, 1971.

[2] A. Iserles, "Exact and discretized stability of the pantograph equation," Applied Numerical Mathematics, vol. 24, no. 2-3, pp. 295-308, 1997.

[3] K. Guan and Z. Luo, "Stability results for impulsive pantograph equations," Applied Mathematics Letters, vol. 26, no. 12, pp. 1169-1174, 2013.

[4] A. Iserles, "On the generalized pantograph functional-differential equation," European Journal of Applied Mathematics, vol. 4, no. 1, pp. 1-38, 1993.

[5] Z. Fan, M. Liu, and W. Cao, "Existence and uniqueness of the solutions and convergence of semi-implicit Euler methods for stochastic pantograph equations," Journal of Mathematical Analysis and Applications, vol. 325, no. 2, pp. 1142-1159, 2007.

[6] Z. Fan, M. Song, and M. Liu, "The $\alpha$ th moment stability for the stochastic pantograph equation," Journal of Computational and Applied Mathematics, vol. 233, no. 2, pp. 109-120, 2009.

[7] H. Zhang, Y. Xiao, and F. Guo, "Convergence and stability of a numerical method for nonlinear stochastic pantograph equations," Journal of the Franklin Institute, vol. 351, no. 6, pp. 3089-3103, 2014

[8] H. Yang, Z. Yang, P. Wang, and D. Han, "Mean-square stability analysis for nonlinear stochastic pantograph equations by transformation approach," Journal of Mathematical Analysis and Applications, vol. 479, no. 1, pp. 977-986, 2019.

[9] W. Xu, W. Xu, and S. Zhang, "The averaging principle for stochastic differential equations with Caputo fractional derivative," Applied Mathematics Letters, vol. 93, pp. 79-84, 2019.

[10] S. Ma and Y. Kang, "Periodic averaging method for impulsive stochastic differential equations with Lévy noise," Applied Mathematics Letters, vol. 93, pp. 91-97, 2019.

[11] B. Pei, Y. Xu, and J.-L. Wu, "Stochastic averaging for stochastic differential equations driven by fractional Brownian motion and standard Brownian motion," Applied Mathematics Letters, vol. 100, Article ID 106006, 2020.

[12] D. Luo, Q. Zhu, and Z. Luo, "An averaging principle for stochastic fractional differential equations with time-delays," Applied Mathematics Letters, vol. 105, Article ID 106290, 2020.

[13] H. M. Ahmed and Q. Zhu, "The averaging principle of Hilfer fractional stochastic delay differential equations with Poisson jumps," Applied Mathematics Letters, vol. 112, Article ID 106755, 2021.

[14] W. Mao, L. Hu, and X. Mao, "The existence and asymptotic estimations of solutions to stochastic pantograph equations with diffusion and Lévy jumps," Applied Mathematics and Computation, vol. 268, pp. 883-896, 2015.

[15] L. Hu, Y. Ren, and Q. He, "Pantograph stochastic differential equations driven by G-Brownian motion," Journal of Mathematical Analysis and Applications, vol. 480, no. 1, Article ID 123381, 2019. 